\title{
Interactions between Asaia, Plasmodium and Anopheles: new insights into mosquito symbiosis and implications in Malaria Symbiotic Control
}

\author{
Aida Capone ${ }^{1 \dagger}$, Irene Ricci ${ }^{1 \dagger}$, Claudia Damiani ${ }^{1}$, Michela Mosca ${ }^{1}$, Paolo Rossi ${ }^{1}$, Patrizia Scuppa ${ }^{1}$, Elena Crotti ${ }^{2}$, \\ Sara Epis ${ }^{1,3}$, Mauro Angeletti ${ }^{1}$, Matteo Valzano ${ }^{1}$, Luciano Sacchi ${ }^{4}$, Claudio Bandi ${ }^{3}$, Daniele Daffonchio ${ }^{2}$, \\ Mauro Mandrioli ${ }^{5}$ and Guido Favia ${ }^{{ }^{*}}$
}

\begin{abstract}
Background: Malaria represents one of the most devastating infectious diseases. The lack of an effective vaccine and the emergence of drug resistance make necessary the development of new effective control methods. The recent identification of bacteria of the genus Asaia, associated with larvae and adults of malaria vectors, designates them as suitable candidates for malaria paratransgenic control.

To better characterize the interactions between Asaia, Plasmodium and the mosquito immune system we performed an integrated experimental approach.
\end{abstract}

Methods: Quantitative PCR analysis of the amount of native Asaia was performed on individual Anopheles stephensi specimens. Mosquito infection was carried out with the strain PbGFPCON and the number of parasites in the midgut was counted by fluorescent microscopy.

The colonisation of infected mosquitoes was achieved using GFP or DsRed tagged-Asaia strains.

Reverse transcriptase-PCR analysis, growth and phagocytosis tests were performed using An. stephensi and Drosophila melanogaster haemocyte cultures and DsRed tagged-Asaia and Escherichia coli strains.

Results: Using quantitative PCR we have quantified the relative amount of Asaia in infected and uninfected mosquitoes, showing that the parasite does not interfere with bacterial blooming. The correlation curves have confirmed the active replication of Asaia, while at the same time, the intense decrease of the parasite.

The 'in vitro' immunological studies have shown that Asaia induces the expression of antimicrobial peptides, however, the growth curves in conditioned medium as well as a phagocytosis test, indicated that the bacterium is not an immune-target.

Using fluorescent strains of Asaia and Plasmodium we defined their co-localisation in the mosquito midgut and salivary glands.

Conclusions: We have provided important information about the relationship of Asaia with both Plasmodium and Anopheles. First, physiological changes in the midgut following an infected or uninfected blood meal do not negatively affect the residing Asaia population that seems to benefit from this condition. Second, Asaia can act as an immune-modulator activating antimicrobial peptide expression and seems to be adapted to the host immune response. Last, the co-localization of Asaia and Plasmodium highlights the possibility of reducing vectorial competence using bacterial recombinant strains capable of releasing anti-parasite molecules.

Keywords: Asaia, Plasmodium, Anopheles, Malaria, Haemocytes

\footnotetext{
*Correspondence: guido.favia@unicam.it

${ }^{\dagger}$ Equal contributors

'Scuola di Bioscienze e Biotecnologie, Università degli Studi di Camerino,

Camerino 62032, Italy

Full list of author information is available at the end of the article
} 


\section{Background}

Malaria represents one of the most devastating infectious diseases with an estimated annual incidence of around 200-500 million cases and 1-3 million deaths per year. Several factors contribute to the severity and worsening of this disease, among which the emergence of drug-resistant parasites and insecticide-resistant mosquitoes are of enormous impact [1]. Malaria parasites (Plasmodium spp.) accomplish part of their biological cycle inside the mosquito vector (Anopheles spp.) following a complex developmental program. A major bottleneck in the Plasmodium spp. cycle occurs in the midgut of mosquitoes, where gametocytes develop into ookinetes and then oocysts: from thousands of gametocytes ingested with a blood meal, only a few oocysts (usually less than 5) will develop [2-4], with some variations depending on the mosquito species as it is well documented in a comparative study in Tanzania focusing on An gambaie s.l. and An. funestus mosquitoes infected with $P$. falciparum [5]. This major bottleneck in the Plasmodium life cycle suggests that the ookinete/oocyst stage represents a target for the development of novel strategies for malaria control. Microorganisms associated with the midgut of mosquitoes might be applied to block the Plasmodium life cycle, and laboratory evidence has already been obtained supporting this possibility [6]. In addition, further stages along the Plasmodium development life-cycle might be targeted for malaria control, i.e. sporozoites after their emergence from oocysts, during their migration, and in the salivary glands. A strategy to exploit symbiotic microorganisms for the control of vector-borne diseases is paratransgenesis, i.e. the generation of engineered symbionts expressing anti-parasite molecules [7]. Different types of microorganisms have already been tested within laboratory conditions for their ability to block malaria parasites, through to the expression of a variety of effect or molecules $[8,9]$. The ideal candidate for the paratransgenesis control of malaria would be a microorganism that has a steady association with Anopheles mosquitoes, infecting not only the midgut but also other tissues where Plasmodium development occurs. This organism should also be amenable to cultivation, genetic manipulation and reintroduction into the mosquito populations. We have recently identified Asaia as a dominant symbiont of some malaria vectors $[10,11]$, and have shown that this symbiont possesses most of the characteristics required for paratransgenesis applications against Plasmodium spp., however, major issues are still to be addressed in order to achieve a better understanding of the Anopheles-Asaia symbiosis. For example, Plasmodium-infected blood meals induce an immune response in mosquitoes that might limit not only the malaria parasite progression in the insect but also the symbiont population. Thus, the first question is whether
Plasmodium interferes with Asaia presence/multiplication in mosquitoes. A related question is whether mosquito immune responses are effective on Asaia. Furthermore, in general, it is important to map the points of overlapping of the parasite (Plasmodium) and the symbiont (Asaia) in the mosquito body.

Using the malaria model An. stephensi-P. berghei, we show that the malaria parasite does not interfere with the growth of Asaia in mosquitoes and that the adaptation of Asaia to the insect immunity might explain its persistence even in mosquitoes heavily infected by the parasite. At the same time, Asaia is widely distributed in both the midgut and the salivary glands and sometimes it is even located in close association with the parasite at different stages of development in the mosquito. These characteristics of Asaia reinforce its potential for paratransgenic applications of malaria control.

\section{Methods}

\section{Mosquitoes}

An. stephensi samples were acquired from a colony reared since 1988 in the insectary of the School of Biosciences and Biotechnology (SBB) at the University of Camerino (Unicam), Italy. These mosquitoes were maintained in standard rearing conditions at a temperature of $29^{\circ} \mathrm{C}$ and $95 \pm 5 \%$ humidity, with a photoperiod of $12 \mathrm{~h}$ light/dark cycle. Adults were fed on mouse blood and a sterilized $5 \%$ sucrose solution.

\section{Malaria parasite}

The malaria parasite used in the experimental infections was the recombinant strain $\mathrm{PbGFP}_{\mathrm{CON}}$, a GFP-tagged recombinant strain derived from the murine pathogenic plasmodia P. berghei ANKA (kindly provided by Sinden R., Imperial College, London, UK). Strain $\mathrm{PbGFP}_{\mathrm{CON}}$ constitutively expresses GFP at higher levels throughout the complete life cycle from a transgene that is integrated into the parasite genome [12]. The usage of $\mathrm{PbGFP}_{\mathrm{CON}}$ allows visualization and investigation of live parasites at various stages of development in both mice and mosquitoes by fluorescent microscopy.

\section{Mice}

Female BALB/c mice were maintained in the breeding facilities of the SBB at Unicam. Eight-week-old female mice were infected with $P$. berghei strain $\mathrm{PbGFP}_{\mathrm{CON}}$ by acyclic passages through an intraperitoneal injection of blood from the tail vein of an infected mouse with around 10\% parasitemia. About $5 \mu$ l of infected blood ( $21 \times 10^{6}$ infected erythrocytes) was diluted in $100 \mu \mathrm{l}$ of $1 \times$ phosphate-buffered saline (PBS) at $\mathrm{pH}$ 7.2. Infected mice were monitored every couple of days for parasitemia by fluorescent microscopy as well as gametocytemia evaluation by through Giemsa stained blood smear. 


\section{Ethical statement}

All animal experiments were carried out according the Italian Directive 116 of 10/27/92 on the "use and protection of laboratory animals" and in adherence with the European regulation (86/609) of 11/24/86 (licence no. 125/94A, issued by the Italian Ministry of Health).

\section{Bacterial strains: GFP-Asaia, DsRed-Asaia and DsRed-E. Coli}

Three recombinant bacterial strains expressing fluorescent proteins, GFP (Green Fluorescent protein) or DsRed (Discosoma Red), have been used: GFP-Asaia, DsRed-Asaia and DsRed-Escherichia coli.

The tagged strain GFP-Asaia was obtained from the native strain SF2.1 isolated from $A$. stephensi by cloning a gfp gene cassette in pHM2 plasmid [10] that confers resistance to kanamycin. The same native strain was also modified to obtain the DsRed-Asaia [13], in this case a mini-Tn5 gene cassette containing the dsRed gene was inserted into the bacterial chromosome by conjugation and transposition [14] and it is indefinitely retained without any antibiotic selective pressure.

The recombinant strain DsRed-E. coli DH5alfa pKan (DsRed) contains the dsRed gene expressed under the control of the ribosomal promoter rrnBP1 [15].

\section{Haemocyte cultures}

'In vitro' experiments were performed using mosquito haemocyte cultures isolated from dissected An. stephensi adult females reared at SBB (UniCam) and macrophagelike Drosophila melanogaster embryonic haemocytes, SL2 cells.

\section{Mosquito infection with $P$. berghei and parasite counts} Quantitative analysis experiments were replicated on three different generations of mosquitoes maintained under the same rearing conditions. Each experimental set-up consisted of two cages, each containing a hundred female mosquitoes that seven days after emergence were fed with a blood meal from anaesthetised, infected or uninfected mice respectively. Infected blood meals were performed using mice showing a parasitemia around $10 \%$. Unfed mosquitoes were removed from both cages, and then, the cages were housed in a chamber at $20^{\circ} \mathrm{C}$ and $95 \pm 5 \%$ humidity, as this step is required for parasite development in laboratory conditions. Before and after the blood meal, mosquitoes were fed with a sterilized 5\% sucrose solution. The control mosquitoes were tested just before the blood meal (five individuals per cage). At 24, 48 and 72 hours (h) after the blood meal ten mosquitoes were examined from each cage. The guts of the mosquitoes collected from both cages were dissected immediately after the sampling. Each single gut was placed in a sterile $1.5 \mathrm{ml}$ "non -stick" low retention hydrophobic tube and carefully homogenized in $60 \mu \mathrm{l}$ of sterile $1 \times$ PBS; low retention hydrophobic pipette tips were used to minimize malaria parasite loss during sample preparation. Half of the gut homogenate preparation was used for the parasite count. Ten $\mu \mathrm{l}$ of the gut homogenate was placed onto a slide and covered with micro glass $(18 \times 18 \mathrm{~mm})$. The green early stages, ookinets and oocysts were counted using a fluorescent microscope Axio observer z1 (Zeiss) at 400x or $200 \times$ objectives, in triplicate.

\section{Quantitative PCR (qPCR) detection of native Asaia in An. stephensi}

Quantitative analysis was carried out on the three experimental set-ups described above. Half of the gut homogenated was used for template preparation for qPCR analysis by Taq-Man probes. DNA was extracted from the guts immediately after the dissection by the Blood GenomicPrep Mini Spin Kit (GE Healthcare) and stored at $+4^{\circ} \mathrm{C}$ in Dnase-free water. The DNA amount was determined using a Nanodrop (Thermo Scientific). Duplex PCR amplifications were carried out, each reaction contained $50 \mathrm{ng}$ of template DNA in $2 \times$ PCR Brilliant Multiplex QPCR Master Mix (Agilent, Stratagene), passive reference dye Rox (1:500), two couples of primers (200 nM each) and two Taq-Man probes (200 nM each). Primers and probes used to amplify target sequences of Asaia 16S rRNA and An. stephensi rps7 genes were obtained by Eurofins probe design [16] and a possible interference among sequences was checked by FastPcr program [17].

Bacterial primers and probe specificities were verified by ProbeMatch toll [18]. Sizes of Asaia 16S rRNA and An. stephensi rps7 amplicons were 99 bp and 90 bp respectively. Amplification of the target sequence $A n$. stephensi rps7 allowed the normalization of an amount of Asaia that was estimated as a relative quantity, calculating the gene copy ratio (number of Asaia $16 \mathrm{~S}$ rRNA/ An. stephensi rps7). The probe sequences were labelled with the reporter dyes Hexachloro-Fluorescein-CE Phosphoramidite (HEX) or Cyanine -5 (Cy5) at the 5' end (emission wavelengths $555 \mathrm{~nm}$ and $665 \mathrm{~nm}$ respectively), and the quencher dyes used were Black Hole Quencher -1 (BHQ-1) or Black Hole Quencher -2 (BHQ-2) respectively. Primers and Taq-Man probe sequences were:

Asaia-16S/for 5'TAGCGTTGCTCGGAATGACTGG3', Asaia-16S/rev 5'CGTATCAAATGCAGCCCCAAGG3', Rps7/For 5'AGCAGCAGCAGCACTTGATTTG3', Rps7/Rev 5'TAAACGGCTTTCTGCGTCACCC3', Asaia-16S/probe HEX-5'AAAGGGCGCGTAGGCGGT TTACACA-3' ${ }^{\prime} \mathrm{HQ}_{1}$, Rps7/probe $\mathrm{Cy}_{5}-5^{\prime}$ CTACTGTGCGTCGTGGGAGATG AACGAA-3 ${ }^{\prime} \mathrm{BHQ}_{2}$ 
The amount of amplified targets was measured by the development of standard curves obtained using templates of eight serial dilutions of plasmid preparations of both amplicons (from 2 to $2 \times 10^{-7} \mathrm{ng}$ ). Standard curves had an average correlation coefficient of 0.999 , a slope of -3.447 , with an amplification efficiency of $95 \%$ in the case of Asaia 16S rRNA amplicon, and an average correlation coefficient of 0.996 , a slope of -3.328 , with an amplification efficiency of $99.7 \%$ in the case of rps7. Efficiency, sensitivity and specificity of the singleplex and duplex assays were comparable.

The thermal cycle was carried out under the following conditions: 10 minutes $(\mathrm{min})$ at $95^{\circ} \mathrm{C} \times$ one cycle; 30 seconds (sec) at $95^{\circ} \mathrm{C}, 1 \mathrm{~min}$ at $60^{\circ} \mathrm{C}$ and $30 \mathrm{sec}$ at $72^{\circ} \mathrm{C} \times$ 40 cycles.

\section{Statistical analysis}

The statistical analysis of the amounts of Asaia was estimated by the qPCR assay, which was provided by the use of the Program R [19]. Asaia blooming after an infected or uninfected blood meal was analysed by ANOVA and post-hoc test 'Bonferroni' that uses a parametric analysis of variance (ANOVA/Bonferroni) based on $\log _{10}$ values that follow the Gaussian trend required [20].

The correlation curves between Asaia amounts and the number of parasites counted have been assessed. The amount of parasites was considered as an independent variable (x) and the Asaia relative quantity as a dependent variable (y). Extreme values were estimated in the range of 3 standard deviations and the correlation was obtained using all data points [21]. Ten points were considered a significant population $(n>9)$. Analysis of linear regression was performed by Student-t and Fisher tests.

\section{Mosquito colonization with recombinant Asaia strains}

Regarding Asaia/Plasmodium co-localisation experiments, two cages have been set-up each one containing a hundred adult females fed with sugar solution, to which GFP- or DsRed-Asaia were added. For colonisation with GFP-Asaia, mosquitoes were fed with sugar solution containing $2 \times 10^{8}$ recombinant bacterial cells $/ \mathrm{ml}$ and $100 \mu \mathrm{g} / \mathrm{ml}$ of kanamycin during the first three days after emergence, while colonisation with DsRed-Asaia was achieved in the same way but without any antibiotic selection.

GFP-Asaia and DsRed-Asaia were grown for $24 \mathrm{~h}$ at $30^{\circ} \mathrm{C}$ in GLY medium, cells were harvested by centrifugation, washed three times in $0.9 \%$ (wt/vol) $\mathrm{NaCl}$ and adjusted to $10^{8}$ cells per $\mathrm{ml}^{-1}$ in $30 \mathrm{ml}$ of $\mathrm{H}_{2} \mathrm{O} / 5 \%$ (wt/ vol) sucrose solution.

Seven days after emergence, the mosquitoes were provided with a blood meal from the same infected mouse in both cages, according to the same protocols previously described for quantitative analysis. Regarding the Asaia/Plasmodium follow up, five mosquitoes from each cage were collected for the dissection of midgut and salivary glands every two days until the 19th day after the blood meal. Midguts were fixed in 4\% paraformaldehyde for $10 \mathrm{~min}$ at $4^{\circ} \mathrm{C}$, whereas salivary glands were analysed on freshly prepared slides to avoid tissue deterioration. The slides were then mounted in glycerol-PBS for analysis by fluorescent microscopy using an Axio observer z1 (Zeiss).

Experiments were performed in duplicate for each recombinant strain.

\section{Semi-quantitative analysis by Reverse Transcription PCR (RT-PCR) of antimicrobial peptide (AMPs) expression 'in vitro'}

In relation to the induction of AMPs expression after bacterial challenge, An. stephensi and D. melanogaster immunocyte cultures were incubated with a $10^{9}$ cells $/ \mathrm{ml}$ bacterial suspension (DsRed-tagged Asaia or E. coli) for $0,4,8$ and $12 \mathrm{~h}$. After treatment, cells were centrifuged at $800 \mathrm{~g}$ for $5 \mathrm{~min}$ at room temperature and the supernatant was discarded. Total RNA was extracted from cells using TRI-REAGENT TM (Sigma), following the method described by the supplier. Following extraction, RNA quality and concentration were assessed with a ND-1000 spectrophotometer (NanoDrop, Wilmington, DE, USA). RT-PCR was performed with the Access RTPCR System (Promega), according to the supplier's protocols.

Cytoplasmic actin was amplified as a loading control and PCR reactions (between 15 and 25 cycles) were carried out using the following parameters: annealing temperature $58^{\circ} \mathrm{C}$, annealing time $40 \mathrm{sec}$, elongation time $45 \mathrm{sec}$. Even if a possible influence of immune challenge on actin expression was reported [22], we saw no differences in normalized versus actin results in either case. Concerning the defending and cecropin A, semiquantitative PCR reactions (35 cycles), the following parameters were used: annealing temperature $61^{\circ} \mathrm{C}$, annealing time $60 \mathrm{sec}$, elongation time $30 \mathrm{sec}$. In the case of drosomycin and the gambicin semi-quantitative PCR reactions (30 and 35 cycles respectively), the following parameters were used: annealing temperature $62^{\circ} \mathrm{C}$, annealing time $50 \mathrm{sec}$, elongation time $30 \mathrm{sec}$. The amplification cycle numbers for the different reactions have been empirically defined for each product to examine the linear phase of PCR, during which template input was proportionally related to amplicon output levels.

The following primers have been used for the reaction:

Actin-F 5'AGCAGGAGATGGCCACC3', Actin-R 5'TCCACATCTGCTGGAAGG3', 
Defensin-F 5'GCTATCGCTTTTGCTCTGCT3', Defensin-R 5'CCACTTGGAGAGTAGGTCGC3', Cecropin-F 5'ACATCTTCGTTTTCGTCGCT3', Cecropin-R 5'CTTGTTGAGCGATTCCCAGC3', Drosomycin-F 5'CCGCAGTACCCACTCAATCT3', Drosomycin-R 5'ACTGCAAAGCCAAAACCATC3', Gambicin-F 5'AACCGGAAGGGGCGTTTCGT3', Gambicin-R 5'CGTCTGGCACTGATTAAACC3'

The expression of defensin, cecropin A, drosomycin and gambicin were evaluated after electrophoresis on $1 \%$ agarose gels. Gel documentation was collected using a "Gel Doc XR", digitally evaluated with "Quantity One" (Bio-Rad Lab., Milan, Italy), and normalized to the correspondent signals for cytoplasmic actin. Seven replicates were carried out.

\section{Growth curves and phagocytosis test}

Mosquito haemocytes were isolated from dissected $A n$. stephensi adult females and maintained for $72 \mathrm{~h}$ in Schneider's medium (Sigma, St. Louis, MO, USA), supplemented with heat-inactivated 10\% FBS (Fetal Bovine Serum), 100 units $/ \mathrm{ml}$ penicillin and $100 \mu \mathrm{g} / \mathrm{ml}$ streptomycin, before further analyses. Antibiotics were removed before phagocytosis and growth tests by centrifugation of cells and resuspension in fresh medium without any addition of antibiotics.

Macrophage-like $D$. melanogaster embryonic haemocytes, SL2 cells, were maintained at $25^{\circ} \mathrm{C}$ in Schneider's medium, supplemented with heat-inactivated 10\% FBS.

Recombinant strains of DsRed-tagged E. coli and Asaia were grown in nutrient broth for $48 \mathrm{~h}$ at $37^{\circ} \mathrm{C}$ and $30^{\circ} \mathrm{C}$ respectively.

For growth inhibition tests, cultures of $E$. coli $\left(10^{9}\right.$ cells/ $\mathrm{ml})$ and Asaia $\left(10^{9}\right.$ cells $\left./ \mathrm{ml}\right)$ were heat-killed at $65^{\circ} \mathrm{C}$ for 45 $\mathrm{min}$. The microorganisms were then centrifuged $(4000 \mathrm{rpm}$ for $10 \mathrm{~min}$ ), washed in PBS and suspended in Schneider's medium in order to obtain a final concentration of heatkilled bacteria of $4 \times 10^{8} / \mathrm{ml}$. Suspensions of both bacteria $(4.5 \mathrm{ml})$ were added to mosquito cultivated haemocytes, at the final concentration of $1.8-2 \times 10^{6} / \mathrm{ml}$, and incubated for $24 \mathrm{~h}$ at $26^{\circ} \mathrm{C}$. After incubation, the suspensions were centrifuged and the supernatants filtered. Medium obtained from mosquito cells exposed to bacteria, was tested undiluted and diluted (1:2) against E. coli and Asaia. The antibacterial activity of the conditioned medium, was registered by Biophotometer recorder (Biophotometer-Bonet-Mauri, Isa Biologie, France) for 30 h. Microorganism growth controls were performed in the Schneider's medium.

In the phagocytic tests, haemocyte cultures from both mosquitoes and flies were incubated for 1 and $2 \mathrm{~h}$ in 5 $\mathrm{ml}$ of medium containing bacteria (E. coli or Asaia), and successively, the phagocytosis index was evaluated as the percentage of phagocytic haemocytes showing inside Ds- red labelled bacteria under an "Eclipse 90i" microscope (Nikon). The microscope was equipped with a super high-pressure mercury lamp and connected to a DS cooled camera head "DS-5Mc" regulated by "ACT-2U" software (Nikon). Ten phagocytic test replicated experiments were performed.

\section{Results and discussion}

Quantitative analysis of native Asaia and P. berghei within An. stephensi

The insect midgut is a major site of immune activity against pathogenic microorganisms [23]. In Anopheles mosquitoes, the ingestion of a blood meal carrying higher loads of Plasmodium is known to elicit a strong immune response, that acts both in the lumen (e.g. through the action of AMPs, nitric oxide and other effector molecules) and at the level of the gut wall [24]. The Anopheles midgut microbiota may be negatively affected by this response. Should the presence of a pathogen such as Plasmodium reduce the load of a given mosquito's midgut symbionts, this would limit the utility of the symbiont itself for applications in paratransgensis. Furthermore, deciphering microbe-pathogen interactions offers new perspectives on disease transmission control [25]. Thus, we have investigated the kinetics of amounts of Asaia in mosquitoes, after supplying the insects with Plasmodium-infected or -uninfected blood meals. The experiments were conducted on three generations of mosquitoes using the murine malaria model P. berghei, with $A n$. stephensi as a mosquito vector. The mosquitoes were examined using qPCR for native Asaia quantification. In particular, for each of the three experimental set-ups, 70 guts were sampled: 10 control guts (before blood meal) and 10 infected guts as well as 10 uninfected guts at 24, 48 and $72 \mathrm{~h}$ after the meal.

The bacterial load showed a growth after the blood meal, detectable at $24 \mathrm{~h}$ and increasing during the next two days, reaching about a tenfold quantity compared to the controls $(\mathrm{p}<0.01)$, in both Plasmodium-infected and -uninfected mosquitoes (Figure 1). Statistical analysis of three experimental set-ups revealed comparable trends in the replicates. Since, the amount of bacteria was sensibly changing among different generations similar to that shown in previous work [26], the results were not shown as an average of three replicates and only one representative data set was shown. Bacterial quantities were represented on a logarithmic scale to display highly increasing values (from $\mathrm{t}=0$ to $\mathrm{t}=72 \mathrm{~h}$ ): blooming of Asaia within the $24-72 \mathrm{~h}$ post blood meal was traced (Figure 1A). To focus on the differences between the control and the other samples, the means \pm SEM have also been reported by $\log _{10}$ values (Figure $1 \mathrm{~B}$ ). There is, therefore, evidence that: i) Asaia blooms in the mosquito midgut after blood ingestion and ii) the bacterial 


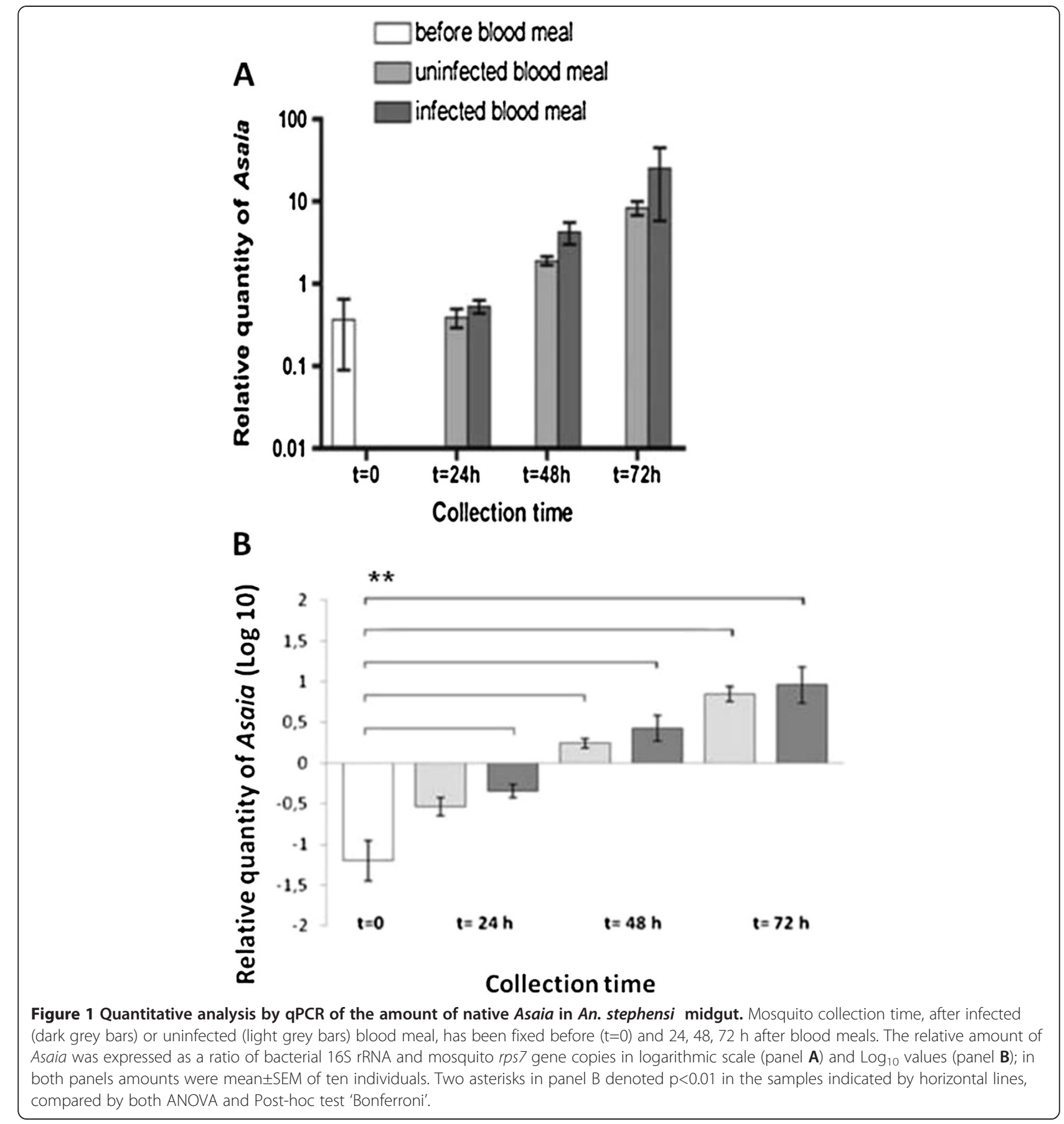

blooming is not affected negatively by the presence of Plasmodium in the blood. We emphasize that the Plasmodium load in infected meals was very high, with about $10 \%$ infected erythrocytes. The growth of bacterial symbionts after the blood meal has already been reported in the case of other arthropods, including mosquitoes [27-29]. However, the evidence that Plasmodium presence at a high load does not interfere with the blooming of a bacterial symbiont (i.e. Asaia) is a novel result. Thus, the immune reaction triggered by
Plasmodium does not interfere with the presence of Asaia in the insect. In summary, parasite infection in the mosquito does not seem to modify the kinetics of Asaia populations after the blood meal: the amount of bacteria in the midgut of infected mosquitoes can be considered at least comparable to that of uninfected specimens, and in considering all the cases, it is higher than in the control group.

In order to explain the quantitative analysis data in more detail, the number of parasites in the infected guts 
at 24,48 , and 72 hour post blood meal has been used to set up the correlation curves of Asaia and Plasmodium loads (Figure 2). Our data showed a drop in Plasmodium infection intensity during Asaia replication in the time period analysed: while the average parasite numbers dropped from $270(24 \mathrm{~h})$, to $134(48 \mathrm{~h})$ and eventually to $94(72 \mathrm{~h})$, there was an approximately eight-fold increase of Asaia at $72 \mathrm{~h}$ compared to $48 \mathrm{~h}$ (slopes ratio $0.33 \times$ / $0.043 \times=7.67$ ). Furthermore, the comparison between the amount of Asaia and the number of parasites in single guts revealed a positive correlation at $48 \mathrm{~h}$ and $72 \mathrm{~h}$ after Plasmodium challenge $(\mathrm{p}<0.05)$, obtaining similar correlation curves $\left(\mathrm{R}^{2}=0.52\right.$ and $\left.\mathrm{R}^{2}=0.53\right)$.

Even though different phenomena could determine this correlation, we might perhaps suggest that the conditions in the mosquito midgut environment, that are more permissive for the development of Plasmodium oocyst, are also favourable for Asaia multiplication. Interestingly, these data showed that experimental infections leading even to one hundred oocysts, as is typical of the model used [30], are accompanied by conspicuous blooming of Asaia. This evidence, reasonably suggests a full compatibility of these two microorganisms for possible paratransgenic applications in nature, in fact, as it is known, the number of $P$. berghei oocysts in $A n$. stephensi tends to be higher than as it is commonly observed in human Plasmodium infections, considering An. gambiae that rarely produce more than two-five oocysts $[4,5]$.

\section{Interaction between Asaia and the mosquito immune system}

The evidence that the ingestion of a blood meal carrying a high load of Plasmodium does not interfere with Asaia growth suggests that this bacterium is not affected by the immune reaction of the mosquito triggered by a malaria parasite challenge. Thus, we performed a series of experiments, with the final goal of determining whether Asaia is resistant to AMPs and phagocytosis. The expression of four AMPs (defensin, cecropin, gambicin and drosomycin) was investigated by semiquantitative RT-PCR analysis on haemocytes from $A n$. stephensi and D. melanogaster, after stimulation in vitro with Asaia or E. coli (Figure 3). Then, the growth curves of both bacterial strains were determined after exposure to conditioned culture medium of the mosquito haemocytes stimulated for the production of AMPs (Figure 4). Finally, we performed phagocytosis tests with haemocytes from An. stephensi and D. melanogaster in order to determine the efficacy of phagocytosis on Asaia using cells from the two insects in comparison with phagocytosis efficacy on E. coli (Figure 5).

The results of these integrated immunological studies can be summarized as follows: i) Asaia induces a comparable expression of cecropin, gambicin and drosomycin in both mosquito (Figure 3A) and fly (Figure 3B) cells, and the response is comparable to that determined by $E$. coli, and in particular, neither of these bacteria seemed to induce defensin gene expression, which has previously been described in the literature on Gram negative strains [31-33]; ii) Asaia growth is only slightly affected by a medium from a haemocyte culture stimulated for AMPs production, which suggests that these bacteria are, in some way, resistant to the mosquito immune effector molecules; iii) Asaia is less phagocytised than E. coli by both An. stephensi and $D$. melanogaster haemocytes, even though the difference is greater in mosquitoes in respect to flies.

Some differences between the capacity of Asaia and E. coli to induce expression of AMPs in An. stephensi haemocyte cultures could be expected since Asaia is stably associated with mosquitoes while $E$. coli has never been reported as a mosquito-associated bacterium.

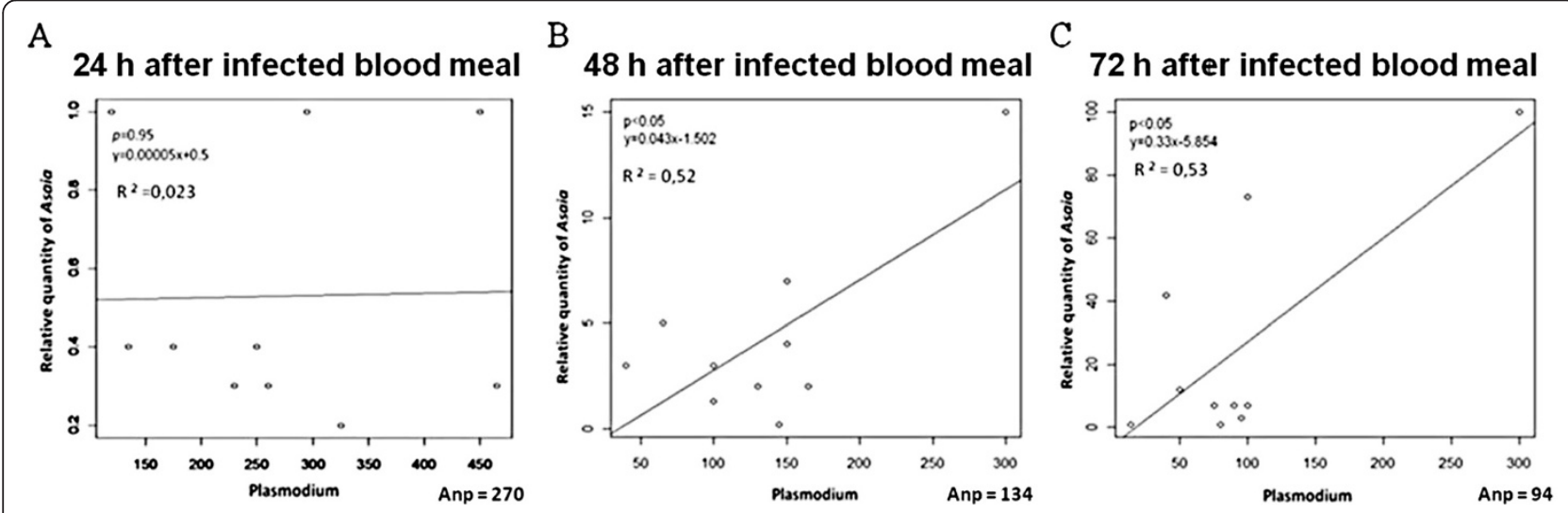

Figure 2 Correlation curves between Asaia and $\boldsymbol{P}$. berghei amounts in An. stephensi midguts. Relative quantities of Asaia obtained from ten individuals presented on a logarithmic scale and the corresponding parasite numbers were reported at 24,48 and $72 \mathrm{~h}$ after the infected blood meal (A, B and $\mathbf{C}$ respectively). The average numbers of parasites (Anp) and the curve slopes ( $\mathrm{Y}$ ) were shown. Analysis of linear regression revealed $p$ values $<0.05$ obtaining a similar correlation curve $\left(R^{2}\right)$ at $48 \mathrm{~h}$ and $72 \mathrm{~h}$. 
A

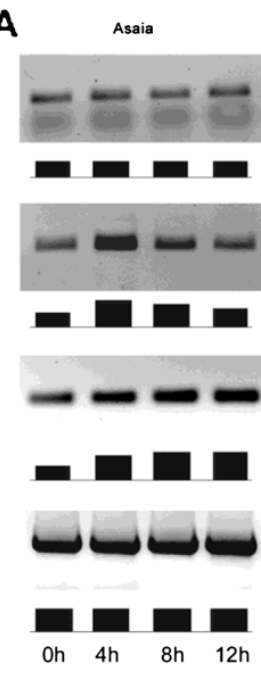

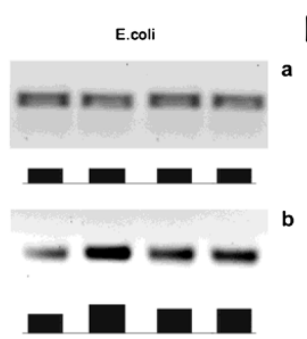
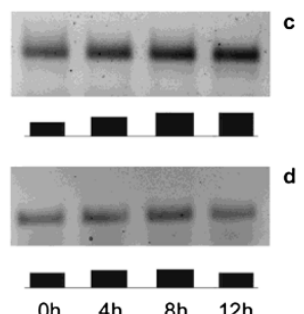

B

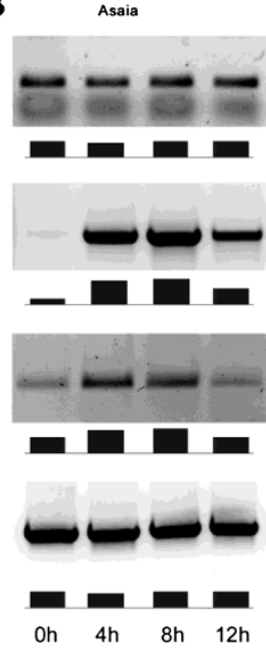

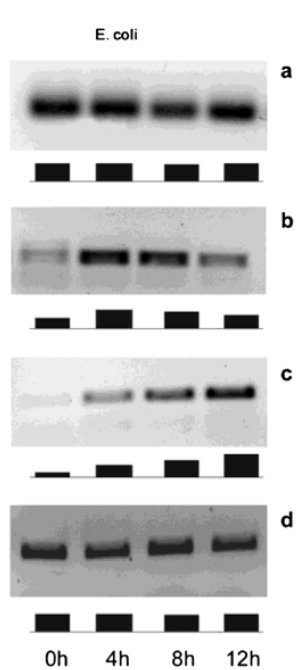

Figure 3 Semi-quantitative analysis by RT-PCR of bacterial induction of AMPs in immunocyte cultures. AMPs expression was evaluated in An. stephensi (panel A) and D. melanogaster (panel B). Defensin (a), cecropin (b), gambicin (c, panel A) and drosomycin (c, panel B) expression at Oh, $4 \mathrm{~h}, 8 \mathrm{~h}$ and $12 \mathrm{~h}$ after Asaia or E. coli bacterial challenge. Actin (d) has been used as a constitutive control gene. The expression of AMPs has been evaluated after electrophoresis in 1\% agarose gel, documentation was collected using a "Gel Doc XR" and digitally evaluated with "Quantity One" as schematized below each panel. One representative set of data out of 7 replicates is shown.

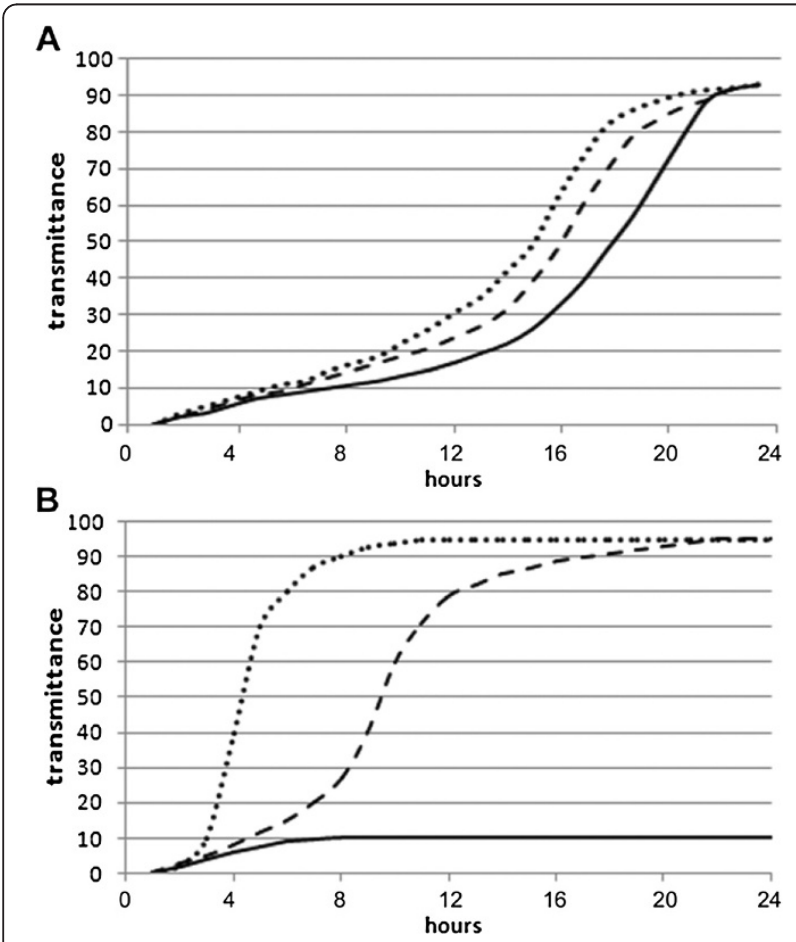

Figure 4 Bacterial growth curves. Asaia (A) and E. coli (B) were cultured in the presence of control Schneider's medium (.....), or halfdiluted $(---)$ and undiluted mosquito haemocytes conditioned medium (-)
Nevertheless, our data support previous evidence showing that Anopheles bacteria induce mosquito antimicrobial immune responses [26]. Even though RT-PCR analysis did not indicate effective secretion of AMPs in the medium, the growth curves suggested the presence of antimicrobial molecules, revealing that $E$. coli multiplication is almost completely inhibited in the undiluted, conditioned medium, and is also partially inhibited in the diluted medium (Figure 4B). Interestingly, under the same growth conditions, Asaia is just slightly and only reduced in the undiluted, conditioned medium (Figure 4A). Therefore, the adaptation (or pre-adaptation) of Asaia to survival within the insect does not appear to be related to a reduced immunogenicity but with resistance to immune responses.

According to this observation, the results of phagocytosis tests also indicate that Asaia is, in some way, adapted to the life within the mosquito: the ratio of $E$. coli/Asaia phagocyting immunocytes was around 2.4 in An. stephensi (Figure 5A) and 1.2 in D. melanogaster (Figure 5B). Thus, mosquito haemocytes are half as effective on Asaia than on E. coli, while the difference using fly haemocytes is not significant (i.e. we might say that the fly haemocytes have almost the same efficacy on the two bacteria). Lastly, haemocytes either in flies or in mosquitoes have shown a similar phagocytic activity of Asaia within a range of $15-20 \%$ (Figure 5).

The results indicating that Asaia is well adapted to resistance to the immune reaction of two different insect species agrees with studies indicating the capacity of these bacteria to colonize phylogenetically distant 


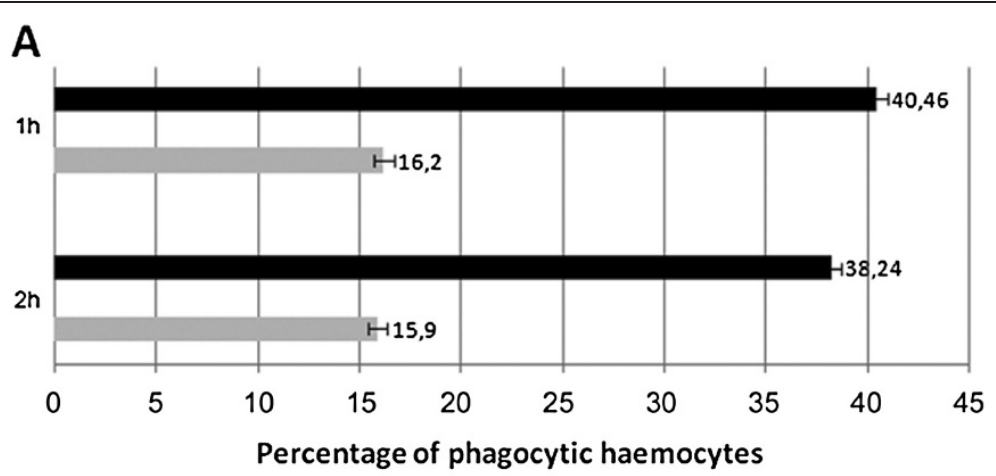

B

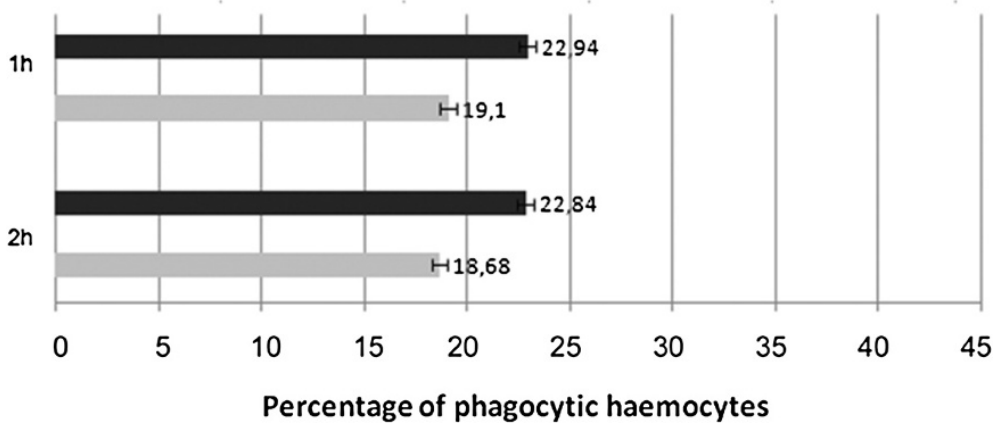

Figure 5 Phagocytosis test analysis. Phagocytic activity was evaluated in vitro using cultured An. stephensi (A) and D. melanogaster (B) haemocytes, and DsRed-labelled bacterial strains of Asaia and E. coli. The percentage of haemocytes showing fluorescent phagocytised bacteria, E. coli (black bars) or Asaia (grey bars), has been evaluated after $1 \mathrm{~h}$ and $2 \mathrm{~h}$. Values are expressed as mean \pm SD of ten replicates.

insects. In addition, the evidence that Asaia is less phagocytised than E. coli by An. stephensi haemocytes (while fly haemocytes have almost the same efficacy on the two bacteria) supports the status of Asaia as a symbiont of anopheline mosquitoes.

\section{Co-localisation of fluorescent strains of Asaia and P. berghei} within An. stephensi midguts and salivary glands

The quantitative analysis results indicated that Plasmodium does not interfere with native Asaia populations within the mosquito gut, even there the bacteria are thriving after the blood meal, usually at the stage of having completed the first part of parasite development (i.e. oocyst formation), that is, at least, comparable to that of uninfected specimens. Both quantitative and in vitro immunological experiments have shown that the host immune response, during the Plasmodium infection, while leading to a drop in parasite number, does not seem to be in conflict with Asaia replication. Then, it was also important to assess whether physiological changes induced by the parasite do not modify the spread of Asaia within the mosquito body. Here we demonstrated that, in infected mosquitoes, Asaia follows the same localization pattern (midgut and salivary glands) as in the absence of the parasite. With this aim, An. stephensi mosquitoes were 'co-infected' with fluorescent-tagged strains of Asaia and P. berghei, and the bacterial spread was monitored in a way as was performed in previous studies on uninfected specimens [10,11,13]. Following emergence, mosquitoes were fed on sugar solution containing one of the two recombinant bacterial strains: GFP-Asaia or DsRedAsaia expressing green or red fluorescent proteins respectively. Then, the mosquitoes colonised with green- or red-labelled bacteria had a blood meal obtained from infected mice harbouring the strain $\mathrm{PbGFP}_{\mathrm{CON}}$ that constitutively expresses the GFP in all the developmental stages of the parasite [12]. The use of two Asaia fluorescent strains, instead of one, allowed us to determine more easily the colonisation pattern within the tissues and to follow the dissemination of bacteria in the mosquitoes. The availability of two recombinant strains was very advantageous: (i) GFPAsaia showed a brighter fluorescent signal compared to the red strain, thus it was more visible in the gut where the presence of very bright oocysts hid the signals of red bacteria; (ii) instead, red-tagged bacteria could be distinguished from sporozoites more easily than the green ones; (iii) the DsRed-Asaia strain allowed a better preservation of the salivary glands (in fact, this tissue became particularly damaged after a prolonged treatment of the mosquitoes with antibiotics).

Microscopic observations revealed fluorescent bacteria and parasites in the midgut showing that Asaia could also be located in close proximity of oocysts (Figure 6). 

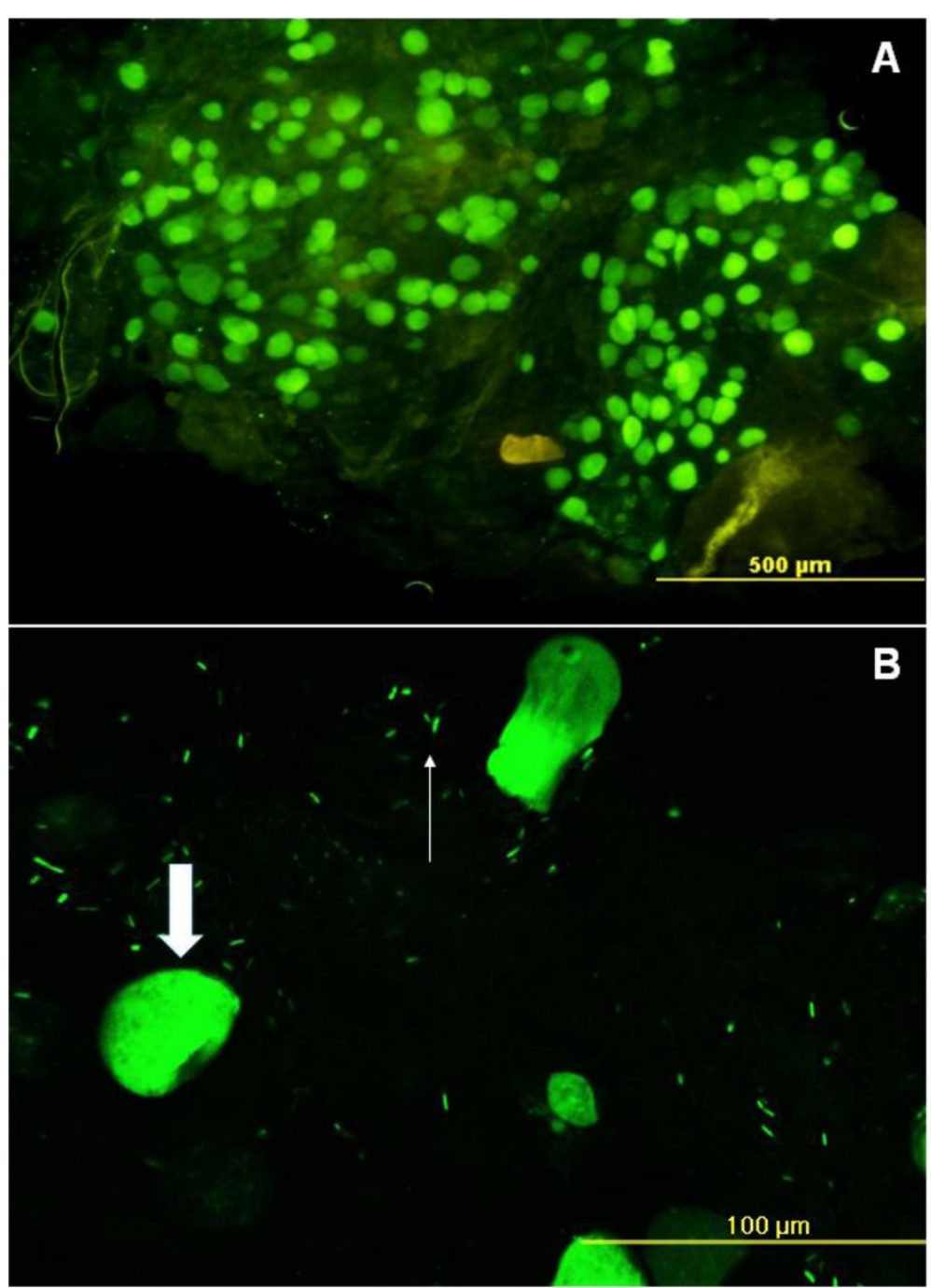

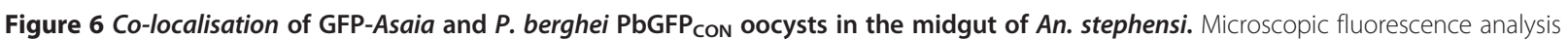
was carried out 11 days after the infected blood meal and at the 15th day after bacterial administration. A massive presence of oocysts is evident in the midgut (A), parasite and bacterial co-localisation is appreciable in the magnified image showing the bacterium (thin arrow) as able to surround and overlap the mature oocysts (thick arrow) (B).

Similarly, fluorescent bacteria surrounded sporozoites that leaked from mature oocysts and were found within the salivary gland lobes, as well (Figure 7). Thus, the presence of Plasmodium does not interfere with the displacement of Asaia that follows the same pattern already observed in uninfected mosquitoes. Furthermore, here we demonstrate that the two microorganisms clearly occupy the same organs where recombinant Asaia strains are widely distributed, and are even able to get in close contact with the parasite. It is a promising enterprise to propose the use of engineered bacteria to release antiparasite molecules within the mosquitoes.

It is an interesting fact that fluorescent Asaia cells were observed to be still present in great numbers within the mosquito organs two-three weeks after bacterial administration (Figures 6 and 7). Microscopy observations, being consistent with quantitative data, revealed that Plasmodium as well as Asaia are able to complete their life cycle or are even capable of multiplication within the mosquitoes. The persistence of a fluorescent signal even after a considerable time after obtaining the marked strains demonstrated the presence of living and replicating bacteria. This observation is in agreement with the results of the immunological experiments (see above) indicating the adaptation of this microorganism to the host immune environment, even in the presence of strong stimulators of immunity, such as Plasmodium.

Moreover, the parasite infection does not seem to interfere with Asaia motility in the mosquito body. 

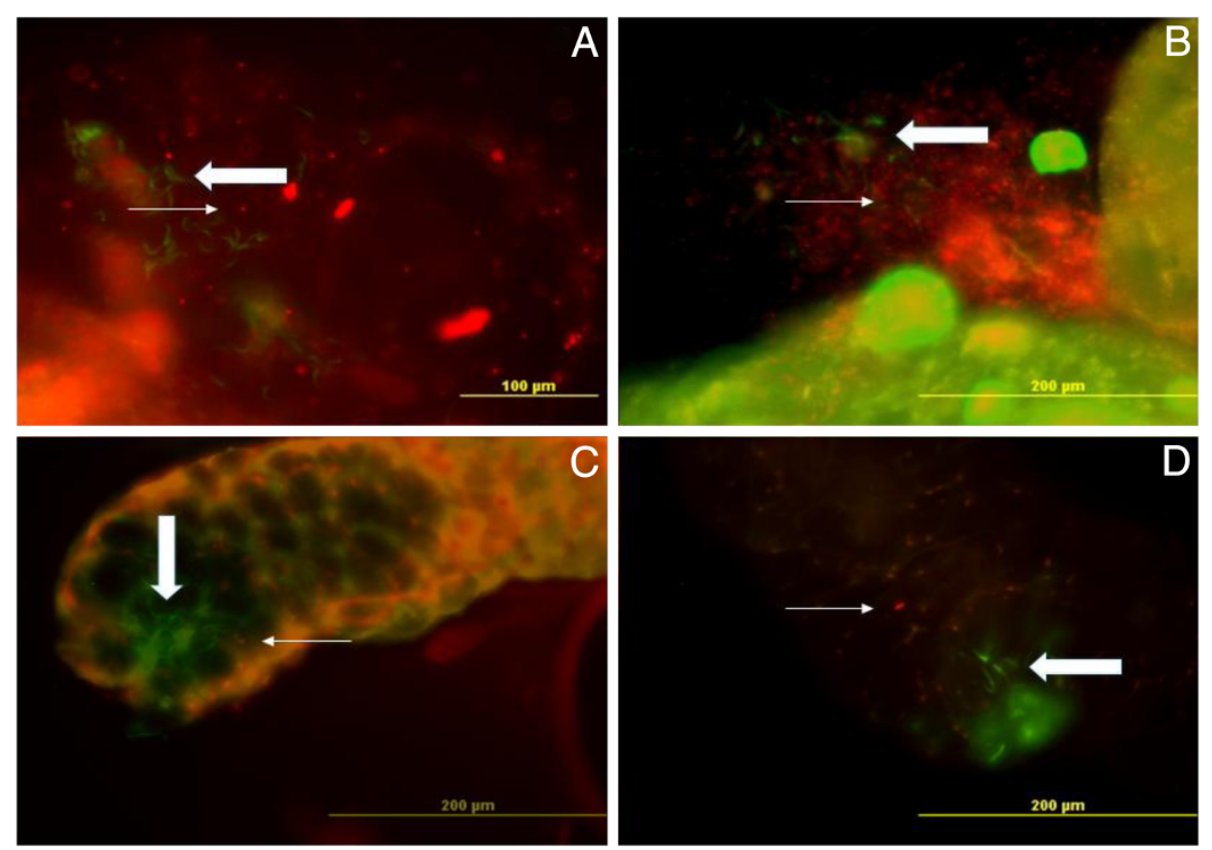

Figure 7 Co-localization of DsRed-Asaia and $P$ berghei PbGFP ${ }_{\text {CON }}$ sporozoites in the proximity of mature oocysts and within the salivary glands of An. stephensi. Microscopic fluorescence analysis was carried out on the 17th day after infection and at the $21 \mathrm{st}$ day after bacterial administration. The presence of red fluorescent Asaia (thin arrow) in the proximity of mature oocysts and GFP-tagged sporozoites (thick arrow) (A and $\mathbf{B}$ ) as well as the co-localisation of the two microrganisms in the salivary gland lobes (C and $\mathbf{D})$ can be detected.

Asaia moves from the crop to other organs, but actually, we cannot yet establish the mechanisms of bacterial translocation. We had already demonstrated that recombinant Asaia strains supplemented by sugar meals are able to colonise the midgut and the salivary glands $[10,11,13]$, however, this had not been demonstrated before in the case of malaria-infected mosquitoes. We have to mention that ookinetes migrate from the midgut lumen to the outer layer of the gut wall thanks to their active motility and molecular interaction with mosquito receptors [34]. Then from this location, sporozoites are released in the hemocoel and they migrate to the salivary glands. Here we showed that these events do not alter the localisation pattern of Asaia.

In conclusion, recombinant strains of Asaia are able: (i) to co-localise with the parasite in the midgut as well as the salivary glands; (ii) to surround Plasmodium in the different stages of development (oocyst and sporozoite); (iii) to preserve their vitality and mobility in infected mosquitoes. All of these characteristics are clearly suitable for paratransgenic approaches.

\section{Conclusions}

In conclusion, if previous works denoted Asaia as a possible paratransgenic candidate for the control of malaria due to its stable association with anopheline mosquitoes, cultivability, transformability and capability of engineered Asaia to colonize mosquitoes by horizontal and vertical transmission routes, the results of this integrated study gathered and presented important information on the relationship of Asaia with both Plasmodium and Anopheles.

First of all, we revealed that physiological changes in the midgut following the blood meal do not negatively affect the residing Asaia population, on the contrary, the benefits of this condition were demonstrated by the bacterial blooming that occurred three days after feeding. Furthermore, the presence of Plasmodium in the blood meal does not interfere with the amounts of bacteria, allowing for the hypothesis that Asaia is able to evade the mosquito immune-response to the parasite infection, even under experimental conditions. This finding is a quite intriguing considering that naturally infected mosquitoes carry about one tenth of the number of oocysts than those reared. This means that, ideally, in wild infected mosquitoes, we would still have a relevant number of Asaia potentially able to exert a paratransgenic action against the parasite. However, when discussing possible implications concerning Malaria Symbiotic Control that arise from these observations, it must be considered that a potential difference between responses to $P$. berghei and $P$. falciparum in An. stephensi could occur just as it has been shown that An. gambiae immune responses to these two parasites are diverse $[35,36]$.

Interestingly, while the parasite number is dropping, the bacterial amount greatly increases over time post- 
infection (8 times more at $72 \mathrm{~h}$ compared to $48 \mathrm{~h}$ ). Nevertheless, we revealed a positive correlation between the number of Asaia and that of Plasmodium in infected mosquitoes, starting from the second day after infection. However, it is possible that this finding could be related to the high infection burden occurring under experimental conditions.

Secondly, we showed that Asaia can act as an immune-modulator microorganism in the mosquito activating the expression of some AMPs, even if it is not affected by these effects or massively phagocytised by haemocytes.

Even if our quantitative data did not find any correlation between the relative amounts of Asaia and Plasmodium within the mosquito midgut at $24 \mathrm{~h}$, after the infected blood meal within the bounds of experimental infections, we cannot rule out that AMPs expression induced by Asaia may interfere with the parasite life cycle during invasion of epithelial tissues and translocation to the salivary glands $[37,38]$; for instance, an enhancement of ookinete lethality by gambicin has been observed [39]. Asaia, as well as other components of the mosquito midgut microbiota, may upregulate some immune genes, including several anti-Plasmodium factors. "Asaiabased" malaria control measure would not be negatively affected by immune reactions played out by the vector mosquito but it could rather reinforce that innate immune response of the mosquitoes.

Lastly, we showed that modified strains of Asaia are widely spread in the midgut and the salivary glands of infected mosquitoes, often being located in proximity of the malaria parasite. Therefore, exogenous recombinant bacterial strains are able to replicate in the mosquito body and to spread in the different organs for two-three weeks after the last administration, thus providing clear evidence that strains of Asaia releasing anti-Plasmodium effector molecules would be able to act against the parasite in the mosquito, reducing its vectorial competence.

All the results presented in this paper highlighted some important features of Asaia that would offer additional support to the feasibility of the Asaia-based malaria control strategies and presented a proof of the concept that attests this potential application in the field.

\footnotetext{
Abbreviations

SBB: School of biosciences and biotechnology; PBS: Phosphate-buffered saline; GFP: Green fluorescent protein; DsRed: Discosoma red; h: Hours; QPCR: quantitative polymerase chain reaction; HEX: Hexachloro-fluoresceinCE phoshoramidite; CY5: Cynine-5; BHQ-1: Black hole quencher-1; BHQ2: Black hole quencher-2; min: Minutes; sec: Seconds; RT-PCR: Reverse transcription polymerase chain reaction; AMPs: Antimicrobial peptides; FBS: Fetal bovine serum; Anp: Average number of parasites.
}

\section{Competing interests}

The authors declare that they have no competing interests.

\section{Authors' contributions}

GF conceived the study and contributed to it with material collection, data analysis, interpretation and manuscript writing. AC, IR, CD, MM, PR, PS, EC, SE, MV, MM, LS provided GPCR and RT-PCR analysis, confocal analysis and immunological studies. MA's contribution was statistical analysis. CB and DD contributed to data interpretation and manuscript writing. All authors read and approved the final version of the manuscript.

\section{Acknowledgements}

We thank M.Q. Benedict and Christophides G.K. for critical reading of the manuscript. $R$. Sinden for providing the PbGFP ${ }_{\text {CON }}$ strain of $P$. berghei. This work was supported by Firb-Ideas (grant RBID082MLZ) and Prin 2009 (grant 009L27YC8_003), both from the Italian Ministry of Education, University and Research (MIUR) and by the EU-FP7 Capacities-Infrastructure 2008 (grant 228421-INFRAVEC) to G.F.

\section{Author details}

${ }^{1}$ Scuola di Bioscienze e Biotecnologie, Università degli Studi di Camerino, Camerino 62032, Italy. ${ }^{2}$ Department of Food, Environmental and Nutritional Sciences, Università degli Studi di Milano, Milan 20133, Italy. ${ }^{3}$ Dipartimento di Patologia Animale, Igiene e Sanità Pubblica Veterinaria, Università degli Studi di Milano, Milan 20133, Italy. ${ }^{4}$ Dipartimento di Biologia Animale, Università degli Studi di Pavia, Pavia 27100, Italy. ${ }^{5}$ Dipartimento di Scienze della Vita, Università degli Studi di Modena e Reggio Emilia, Modena 41125, Italy.

Received: 18 February 2013 Accepted: 12 June 2013

Published: 18 June 2013

\section{References}

1. Shiff C: Integrated approach to malaria control. Clin Microbiol Rev 2002, $15: 278-293$.

2. Sinden RE: Plasmodium differentiation in the mosquito. Parassitologia 1999, 41:139-148.

3. Sinden RE, Alavi Y, Raine JD: Mosquito-malaria interactions: a reappraisal of the concepts of susceptibility and refractoriness. Insect Biochem $\mathrm{Mol}$ Biol 2004, 34:625-629.

4. Vaughan JA: Population dynamics of Plasmodium sporogony. Trends Parasitol 2007, 23:63-70.

5. Taylor LH: Infection rates in, and the number of Plasmodium falciparum genotypes carried by Anopheles mosquitoes in Tanzania. Ann Trop Med Parasitol 1999, 93:659-662.

6. Riehle MA, Moreira CK, Lampe D, Lauzon C, Jacobs-Lorena M: Using bacteria to express and display anti-Plasmodium molecules in the mosquito midgut. Int J Parasitol 2007, 37:595-603.

7. Coutinho-Abreu IV, Zhu KY, Ramalho-Ortigao M: Transgenesis and paratransgenesis to control insect-borne diseases: current status and future challenges. Parasitol Int 2010, 59:1-8

8. Fang W, Vega-Rodríguez J, Ghosh AK, Jacobs-Lorena M, Kang A, St Leger RJ: Development of transgenic fungi that kill human malaria parasites in mosquitoes. Science 2011, 331:1074-1077.

9. Wang S, Ghosh AK, Bongio N, Stebbings KA, Lampe DJ, Jacobs-Lorena M: Fighting malaria with engineered symbiotic bacteria from vector mosquitoes. PNAS USA 2012, 109:12734-12739.

10. Favia G, Ricci I, Damiani C, Raddadi N, Crotti E, Marzorati M, Rizzi A, Urso R, Brusetti L, Borin S, Mora D, Scuppa P, Pasqualini L, Clementi E, Genchi M, Corona S, Negri I, Grandi G, Alma A, Kramer L, Esposito F, Bandi C, Sacchi L, Daffonchio D: Bacteria of the genus Asaia stably associate with Anopheles stephensi, an Asian malarial mosquito vector. Proc Natl Acad Sci USA 2007, 104:9047-9051.

11. Damiani C, Ricci I, Crotti E, Rossi P, Rizzi A, Scuppa P, Capone A, Ulissi U, Epis S, Genchi M, Sagnon N, Faye I, Kang A, Chouaia B, Whitehorn C, Moussa GW, Mandrioli M, Esposito F, Sacchi L, Bandi C, Daffonchio D, Favia G: Mosquito-bacteria symbiosis: the case of Anopheles gambiae and Asaia. Microb Ecol 2010, 60:644-654

12. Franke-Fayard B, Trueman H, Ramesar J, Mendoza J, van der-Keur M, van der-Linden R, Sinden RE, Waters AP, Janse CJ: A Plasmodium berghei reference line that constitutively expresses GFP at a high level throughout the complete life cycle. Mol Biochem Parasitol 2004, 137:23-33. 
13. Damiani C, Ricci I, Crotti E, Rossi P, Rizzi A, Scuppa P, Esposito F, Bandi C, Daffonchio D, Favia G: Paternal transmission of symbiotic bacteria in malaria vectors. Curr Biol 2008, 18:1087-1088.

14. Mølbak L, Molin S, Kroer N: Root growth and exudate production define the frequency of horizontal plasmid transfer in the Rhizosphere. FEMS Microbiol Ecol 2007, 59:167-176.

15. Crotti E, Damiani C, Pajoro M, Gonella E, Rizzi A, Ricci I, Negri I, Scuppa P, Rossi P, Ballarini P, Raddadi N, Marzorati M, Sacchi L, Clementi E, Genchi M, Mandrioli M, Bandi C, Favia G, Alma A, Daffonchio D: Asaia, a versatile acetic acid bacterial symbiont, capable of cross-colonizing insects of phylogenetically distant genera and orders. Environ Microbiol 2009, 11:3252-3264

16. Eurofins probe design. www.eurofinsdna.com.

17. FastPcr program. http://primerdigital.com/fastpcr.html.

18. ProbeMatch toll. http://rpd.cme.msu.edu/probematch/search.jsp.

19. Program R. http://cran.r-project.org/bin/,

20. Box GEP, Cox DR: An analysis of transformations. In Journal of the Royal Statistical Society Volume 26. 2nd edition. 1964:211-252.

21. Anscombe FJ: Rejection of outliers. In American Society for Quality Volume 2. 2nd edition. ; 1960:123-147.

22. de-Morais GS, Vitorino R, Domingues R, Tomer K, Correia AJ, Amado F, Domingues P: Proteomics of immune-challenged Drosophila melanogaster larvae haemolymph. Biochem Biophys Res Commun 2005, 328:106-115.

23. Kumar S, Barillas-Mury C: Ookinete-induced midgut peroxidases detonate the time bomb in anopheline mosquitoes. Insect Biochem Mol Biol 2005, 35:721-727.

24. Christophides GK, Vlachou D, Kafatos FC: Comparative and functional genomics of the innate immune system in the malaria vector Anopheles gambiae. Immunol Rev 2004, 198:127-148.

25. Boissier A, Tchioffo MT, Bachar D, Abate L, Marie A, Nsango SE, Shahbazkia HR, Awono-Ambene PH, Levashina EA, Christen R, Morlais I: Midgut microbiota of the malaria mosquito vector Anopheles gambiae and interactions with Plasmodium falciparum infection. PLoS Pathog 2012, 8:e1002742.

26. Dong Y, Manfredini F, Dimopoulos G: Implication of the mosquito midgut microbiota in the defense against malaria parasites. PLOS Pathog 2009, 5:e1000423.

27. Pumpuni CB, Demaio J, Kent M, Davis JR, Beier JC: Bacterial population dynamics in three anopheline species: the impact on Plasmodium sporogonic development. AmJTrop Med Hyg 1996, 54:214-218.

28. Eichler S, Schaub GA: Development of symbionts in triatomine bugs and the effects of infections with trypanosomatids. Exp Parasitol 2002, 100:17-27.

29. Zayed ME, Bream AS: Biodiversity of the microbial flora associated with two strains of Culex pipiens (Diptera: Culicidae). Commun Agric Appl Biol Sci 2004, 69:229-234.

30. Sinden RE, Dawes EJ, Alavi Y, Waldock J, Finney O, Mendoza J, Butcher GA, Andrews L, Hill AV, Gilbert SC, Basáñez MG: Progression of Plasmodium berghei through Anopheles stephensi is density-dependent. PLOS Pathog 2007, 3:e195.

31. Imler JL, Bulet P: Antimicrobial peptides in Drosophila: structures, activities and gene regulation. Chem Immunol Allergy 2005, 86:1-21.

32. Bulet $P$, Stöcklin R: Insect antimicrobial peptides: structures, properties and gene regulation. Protein Pept Lett 2005, 12:3-11.

33. Lemaitre B, Hoffmann J: The host defense of drosophila melanogaster. Annu Rev Immunol 2007, 25:697-743.

34. Vlachou D, Schlegelmilch T, Runn E, Mendes A, Kafatos FC: The developmental migration of Plasmodium in mosquitoes. Curr Opin Genet Dev 2006, 16:384-391.

35. Dong Y, Aguilar R, Xi Z, Warr E, Mongin E, Dimopoulos G: Anopheles gambiae immune responses to human and rodent Plasmodium parasite species. PLoS Pathog 2006, 2:e52.

36. Mendes AM, Awono-Ambene PH, Nsango SE, Cohuet A, Fontenille D, Kafatos FC, Christophides GK, Morlais I, Vlachou D: Infection intensitydependent responses of Anopheles gambiae to the African malaria parasite Plasmodium falciparum. Infect Immun 2011, 79:4708-4715.
37. Dimopoulos G, Seeley D, Wolf A, Kafatos FC: Malaria infection of the mosquito Anopheles gambiae activates immune-responsive genes during critical transition stages of the parasite life cycle. EMBO J 1998, 17:6115-6123.

38. Dimopoulos G, Casavant TL, Chang S, Scheetz T, Roberts C, Donohue M, Schultz J, Benes V, Bork P, Ansorge W, Soares MB, Kafatos FC: Anopheles gambiae pilot gene discovery project: identification of mosquito innate immunity genes from expressed sequence tags generated from immune-competent cell lines. Proc Natl Acad Sci USA 2000, 97:6619-6624.

39. Vizioli J, Bulet P, Hoffman JA, Kafatos FC, Muller HM, Dimopoulos G: Gambicin: a novel immune responsive antimicrobial peptide from the malaria vector Anopheles gambiae. Proc Natl Acad Sci USA 2001, 98:12630-12635.

\section{doi:10.1186/1756-3305-6-182}

Cite this article as: Capone et al:: Interactions between Asaia,

Plasmodium and Anopheles: new insights into mosquito symbiosis and implications in Malaria Symbiotic Control. Parasites \& Vectors 2013 6:182

\section{Submit your next manuscript to BioMed Central and take full advantage of:}

- Convenient online submission

- Thorough peer review

- No space constraints or color figure charges

- Immediate publication on acceptance

- Inclusion in PubMed, CAS, Scopus and Google Scholar

- Research which is freely available for redistribution

Submit your manuscript at www.biomedcentral.com/submit
C Biomed Central 